\section{La Révolution française}

Cahiers de l'Institut d'histoire de la Révolution française

5 | 2013

Le républicanisme anglais dans la France des

Lumières et de la Révolution

\title{
D'une révolution à l'autre : Alphonse Aulard face aux événements russes de 1917
}

\section{Antonino de Francesco}

\section{(2) OpenEdition}

\section{Journals}

Édition électronique

URL : http://journals.openedition.org//rf/986

DOI : $10.4000 /$ Irf.986

ISSN : 2105-2557

Éditeur

IHMC - Institut d'histoire moderne et contemporaine (UMR 8066)

Référence électronique

Antonino de Francesco, «D'une révolution à l'autre : Alphonse Aulard face aux événements russes de 1917 », La Révolution française [En ligne], 5 | 2013, mis en ligne le 31 décembre 2013, consulté le 10 décembre 2020. URL : http://journals.openedition.org/lrf/986 ; DOI : https://doi.org/10.4000/lrf.986

Ce document a été généré automatiquement le 10 décembre 2020.

(c) La Révolution française 


\title{
D'une révolution à l'autre : Alphonse Aulard face aux événements russes de 1917
}

\author{
Antonino de Francesco
}

1 Dans un curieux petit livre de souvenirs de jeunesse, le franco-argentin René Sidersky, qui fut l'élève d'Alphonse Aulard à Paris au début du XXe siècle, trouve à maintes reprises le moyen de rappeler les querelles scientifico-culturelles qui traversèrent à cette époque les études révolutionnaires. Dur à l'égard de Mathiez, qui, selon lui, ne s'était opposé à leur maître commun que pour plus rapidement le remplacer dans l'enseignement universitaire à la Sorbonne, peu intéressé par la figure de Sagnac (dont les travaux lui semblaient de peu d'envergure), Sidersky est en revanche fasciné par Georges Lefebvre, dont il rappelle le caractère certes sectaire, mais dont il reconnaît la qualité de l'enseignement scientifique. Curieux témoignage que celui-ci : qui suggère que, à la veille de 1968, au moment de livrer son bref manuscrit à l'impression, Sidersky, tout en étant partisan d'Aulard, reconnaissait le caractère dominant d'une autre orientation scientifique et culturelle, autrement dit celle du binome Godechot-Soboul qui, depuis quelques années déjà, avait fait de l'héritage scientifique et culturel de Lefebvre sa propre référence ${ }^{1}$.

2 Les choix de Sidersky en ce début de $\mathrm{XX}^{\mathrm{e}}$ siècle me semblent, de ce point de vue, importants, d'une part pour expliquer l'éclipse rapide d'Aulard du panorama scientifique français de l'entre-deux guerres et d'autre part, pour comprendre comment le fondateur de l'enseignement universitaire de la Révolution française ait pu aussi rapidement disparaître du centre d'intérêt des études pour la génération suivante. A y regarder de plus près, il s'agit là d'un déclin rapide et apparemment irréversible : déjà, dès le début des années 30, l'orientation historiographique de Mathiez avait désormais pris le dessus, aussi bien au niveau international qu'en France même, où les disciples d'Aulard rencontraient les plus grandes difficultés à maintenir non seulement en vie son approche historiographique mais surtout, sa position politique ${ }^{2}$. 
On sait en effet, que les deux adversaires, tous deux favorables en 1914 à la guerre contre l'Allemagne, furent rapidement amenés à se diviser quant aux choix politiques de l'immédiat après-guerre : Aulard, se maintenant dans le cadre de la tradition radicale de la III République; Mathiez s'en éloignant très tôt pour forger, à travers sa prise de position de 1920 sur la cohérence entre jacobinisme et bolchevisme, un mode profondément différent de vivre politiquement l'étude de la révolution française : l'abord avec l'œuvre de Jaurès, ajouté à l'idée que l'octobre de Lénine était un nouveau 1793, reléguait définitivement au rang des vieilleries l'œuvre historiographique d'Alphonse Aulard.

Qu'il s'agisse là d'un conflit politique quant à la manière de définir et de vivre la gauche française, en témoigne la nécrologie que l'Humanité, organe officiel du parti communiste, consacra à Aulard. Lisons-la presque intégralement :

[Aulard] ... s'était spécialisé dans l'étude de la Révolution française. Mais il a luimême dit, après la parution de l'histoire de Jaurès, que ce magnifique travail annulait ses propres travaux. De fait, alors que le grand militant socialiste s'est efforcé de comprendre la Révolution en étudiant son infrastructure économique, Aulard s'était cantonné dans de puériles « explications » personnelles. Il s'affirmait «dantonien » et l'histoire de l'extraordinaire éruption populaire de 1789-1793 ne lui apparaissait que comme un prétexte à faire « un grand homme ». De bons esprits lui ont justement reproché d'avoir truqué les faits au bénéfice de son « héros » et d'avoir calomnié Robespierre, symbole de la dictature prolétarienne. Aulard était en politique un honnête radical droitdel'homard. C'est-à-dire qu'il déformait volontiers l'histoire au bénéfice de son parti. Il protesta cependant contre les sottises de Béraud sur la Révolution russe. Mais il ne sut que trop tard quitter Le Quotidien, dont il couvrit, durant des mois, la politique des fonds secrets et de compromissions louches en compagnie de Renaudel. ${ }^{3}$

5 Ainsi, même en filigrane, la démolition de l'œuvre d'Aulard, par respect pour les orientations historiographiques redéfinies par Mathiez (qu'on ne pouvait pourtant citer parce qu'il était entre-temps sorti du parti), se conjugue à la dénonciation d'une ligne politique désormais dépassée, fondée sur les orientations de la Ligue des Droits de l'Homme, dont Aulard fut toujours magna pars; des choix politiques qui demeuraient résolument antibolchéviques, et qui favorisèrent sa rencontre avec le réformisme socialiste de Pierre Renaudel, à l'image de ce rapprochement de plus grande échelle qui aurait donné lieu à la formation de ce cartel électoral radical-socialiste de 1924.

6 C'est sans doute dans un tel climat, où les études révolutionnaires apparaissaient désormais dominées par Mathiez, que se fit le transfert aux Etats-Unis, dans les années qui suivirent immédiatement la mort d'Aulard, de ses papiers et de sa bibliothèque. De ce transfert, on sait encore peu de choses, mais il semble important de souligner que celui-ci se déroula justement à cheval sur les années 30 : l'intégralité du fonds fut acquise, d'abord à New York par James Buell Munn, professeur d'anglais à ladite université, qui à l'occasion de son retour à Harvard en 1932, voulut en faire le don anonymement à son département. De plus, il s'agit là d'un fonds assez volumineux - constitué essentiellement de 2328 volumes et 1213 pamphlets, déposés à la Widener Library - auxquels il faut ajouter les notes des conférences qu'Aulard donna de 1886 jusqu'à sa retraire en 1922, et qui eux, sont toujours conservés à Harvard, mais auprès de la Houghton Library ${ }^{4}$.

7 En outre, on trouve justement à l'intérieur de ces papiers certains écrits d'Aulard datant de la guerre, dans lesquels il est possible de suivre son positionnement par rapport au conflit mondial et à la Révolution russe, et de définir par conséquent plus précisément 
par rapport à ce qu'on en disait jusqu'alors, de quelle manière et en quels termes il affronta les événements de 1917. Jusqu'alors en effet, la lecture par Aulard de la Révolution russe a été présentée soit en termes ambigus et superficiels ${ }^{5}$, soit sur un mode certainement plus structuré, sous le signe d'un parallèle systématique entre 1917 et 1793 : ce parallèle est celui qui, selon Sergio Luzzato, indique dans les choix d'Aulard une anticipation du discours radical-socialiste sur la Révolution russe - discours entièrement imprégné par l'analogie, toujours et partout recherchée, entre cette révolution et le précédent français ${ }^{6}$. C'est encore cette même interprétation, mais sous un angle différent, que proposait François Furet, quand, faisant référence aux réflexions d'Aulard du début de 1919, il rappelle que la recherche de cette analogie était un réflexe conditionné alors, suffisamment fort pour amener un bon professeur de couleur radicale à accueillir, sans autre forme de procès, ce même Lénine dans le sillage de la tradition révolutionnaire, et à présenter les «journées d'octobre » comme comparables, par exemple à celles du 31 mai et du 2 juin 1793, qui amenèrent la chute de la Gironde et l'instauration du gouvernement révolutionnaire?

Cependant, ces deux lectures, qui insistent beaucoup sur la valeur incantatrice de l'analogie historique, ne sont pas neuves; elles reprennent un argument largement répandu durant ces années, tant en France qu'en Union Soviétique : à partir de 1921, comme on le sait, Raul Labry regrettait l'insistance avec laquelle les intellectuels français lisaient les événements de Russie à l'aune de la Révolution française ${ }^{8}$; peu de temps après, Henri de Man, qui avait fait partie de la délégation socialiste en visite en Russie, insistait sur le fait qu'à leur tour, les protagonistes de 1917 - de février comme d'octobre - agissaient avec la certitude de refaire le chemin de $1793^{\circ}$. Toutefois, s'il est vrai qu'au moins au départ, Aulard fut lui-même tenté par ces analogies si faciles, il est non moins certain que les exemples apportés jusqu'alors à l'appui de cette thèse ne s'appuient que sur quelques moments de l'engagement intellectuel (et politique) d'Aulard et en viennent finalement à polariser sur quelques aspects seulement, une confrontation avec la Révolution russe que l'historien engagea certes très tôt, mais qu'il n'amena à son terme, en y regardant de près, que bien plus tard.

En effet, un certain nombre de notes dactylographiées pour le Journal de Russie, feuille semi-officielle du gouvernement français lancée à l'initiative de l'ambassadeur à Petrograd Albert Thomas, ajoutées à d'autres réflexions, elles aussi conservées parmi les papiers de ses conférences universitaires, permettent en fait d'élargir la perspective et d'avoir une approche plus précise de la lecture par Aulard de la Révolution russe ${ }^{10}$. Nous pouvons en retracer, même brièvement, les étapes. Au départ, il y a une déclaration du 18 mars 1917 émanant de la Société d'histoire de la Révolution française, fondée et animée par Aulard, dans laquelle les événement de février sont salués comme un extraordinaire virage démocratique : mettant sur le même plan 1789 et 1792, la prise de la Bastille et la journée du 10 août, le télégramme de félicitations, bien qu'envoyé à la Douma, constitue une preuve évidente d'une conviction très forte d'Aulard selon laquelle l'extraordinaire changement en cours à l'Est ne pouvait que favoriser une relance, dans un sens démocratique, et en soutien aux nationalités opprimées, de l'effort de guerre de la Russie ${ }^{11}$.

10 Peu de temps après, Aulard aurait reproduit la même règle à l'occasion de la célèbre manifestation de soutien à la Révolution russe organisée le $1^{\text {er }}$ avril à Paris par la Ligue des Droits de l'Homme. A cette occasion, devant un public de plus de 5000 personnes, libre de se réunir pour la première fois depuis le début des hostilités, les choses ne prirent 
pourtant pas la tournure voulue par les organisateurs: plusieurs centaines de manifestants contestèrent à plusieurs reprises les orateurs, au point que la manifestation - après le discours introductif de Victor Basch et les reconstructions historiques de Victor Bérard et d'Alphonse Aulard, après l'exposé passionné du socialiste Vandervelde - dut être soudainement interrompue, en raison de la contestation d'une partie du public qui empêcha le responsable de la CGT, Léon Jouhaux, et le leader de la SFIO, Pierre Renaudel, de prendre la parole ${ }^{12}$.

11 Alphonse Aulard aurait toutefois repris son discours, en le publiant, en France, dans le Bulletin de la Ligue, et dans une version plus développée en Suisse, dans l'intention comme cela sembla par la suite être effectivement le cas - de le faire traduire en russe et de le faire circuler à l'Est ${ }^{13}$. Nous n'avons pas ici la possibilité de confronter dans leur intégralité les deux versions (le texte qu'Aulard lut le $1^{\mathrm{er}}$ avril au cours de la manifestation troublée et qu'il publia dans le Bulletin avec la version définitive publiée à Lausanne le 25 mai) : en nous contentant donc de souligner le très net allongement du texte, il faut au surplus rappeler que le trait commun aux deux travaux réside dans la proposition de mettre en corrélation 1776, 1789 et 1917 afin de mieux mettre en évidence le fait que la Révolution russe fût la descendante directe de la Révolution française, de même que cette dernière l'était de la Révolution américaine; tout en rapprochant les causes de la guerre des précédents historiques, selon Aulard, grâce à ce qui advenait à l'Est, le voyage de la démocratie était parvenu à son terme, précisément parce que la séquence de trois événements révolutionnaires sanctionnait le triomphe définitif de la cause de la démocratie et en conséquence, ouvrait la voie à une paix juste entre les nations. S'empressant de rappeler qu'en Russie, s'était fait en quelques jours ce que les Français avaient au contraire mis trois longues années à réaliser, Aulard, tout en rendant hommage à l'absence de recours à la violence, n'en mettait pas moins en garde contre les risques du modérantisme pour souhaiter la prompte démocratisation de la vie sociale et politique de l'allié qui n'aurait pu avoir lieu qu'au moyen de la fondation d'une République ; et, tout en insistant toujours beaucoup sur les similitudes avec le processus révolutionnaire français, il ne manquait cependant pas de douter de la volonté de la nouvelle Russie de poursuivre la guerre, orientant, par un tel choix, la politique des alliés vers l'objectif de la véritable paix de solidarité entre les nations.

Dès lors, précisément, la conjonction entre révolution et poursuite de la guerre fut à la base des contestations auprès d'un public en majorité socialiste, fatigué au contraire de la guerre ${ }^{14}$. Et pourtant, sur ce point, Aulard, fidèle à ses certitudes personnelles quant au bienfondé de l'interventionnisme, tint toujours ferme. Pour cette raison, il se dit bientôt disposé à écrire aussi pour le Journal de Russie, un journal dont nous avons dit qu'il bénéficiait du soutien ferme de l'ambassade de Paris et qui était animé par l'envoyé spécial en Russie du Temps, Ludovic Naudeau, un journaliste lui aussi interventionniste mais de convictions politiques radicales en tout point semblables à celles d'Aulard ${ }^{15}$. Des critiques sévères ne manquèrent pas de s'élever quant à ce choix de publier un journal politique en langue française en Russie: Raoul Labry, responsable de l'Institut culturel français à Petrograd, rappela, quelques années plus tard, qu'il aurait été nécessaire de le rédiger en russe ; la nécessité de trouver des financements dans la communauté française empêcha cette option qui aurait seule permis une large diffusion des positions françaises auprès de la population russe ${ }^{16}$. Si l'on en croit Naudeau, le journal fut cependant lu par les élites, au point que les bolcheviks eux-mêmes le prirent rapidement en ligne de mire, jusqu'à en interdire la parution en juillet 1918, et à incarcérer son directeur pendant plus 
de quatre mois - avant qu'une lettre d'abjuration de ce dernier, publiée dans la Pravda, ne lui permit de retourner en France ${ }^{17}$.

13 Les articles d'Aulard sont donc intéressants, non seulement pour mesurer ses choix politiques par rapport à octobre, mais aussi pour comprendre les raisons de sa future disgrâce auprès de l'historiographie soviétique. Sa collaboration au Journal de Russie commença précisément juste avant octobre, par un article qui fut publié au lendemain même de la chute de Kerensky, dans lequel - face aux difficultés dans lesquelles se débattait le gouvernement provisoire, Aulard rappelait combien la mise en route et la structure de la Révolution française étaient différentes de celle de Russie: 1789 avait toujours été rigoureusement unitaire, et avait de cette façon fait de la construction d'une seule nation un des principaux motifs de refus de l'Ancien Régime ; à l'inverse, 1917 allait dans la direction opposée, s'orientait vers la construction d'une identité fédérale qui réunissait librement entre elles des nationalités très différentes, et ce projet finissait par compromettre le chemin de la démocratie russe ${ }^{18}$. Suivait un autre article, écrit à Paris le 27 octobre, où la référence à la Révolution française ne se concentrait plus que sur le souvenir du Comité de Salut Public, lequel, en dépit des graves difficultés intérieures, n'avait jamais renoncé à faire la guerre aux puissances réactionnaires, obtenant cette victoire de Fleurus qu'Aulard souhaitait voir sourire au plus vite aux Russes, à qui il rappelait implicitement leurs engagements militaires contractés précédemment ${ }^{19}$. Puis, dans un autre article, écrit le 4 novembre, Aulard insistait sur les raisons justifiant la poursuite de la guerre, en soulignant combien les demandes d'émancipation et de démocratisation étaient compréhensibles et même souhaitables, étant donné que sur le versant français même, à la fin du conflit mondial, les poilus auraient réclamé une démocratisation à venir de la vie politique. Pour cette raison, le lien entre 1776, 1789 et 1917 était réaffirmé pour souligner ensuite l'importance de la naissance de la Société des Nations dans laquelle la Russie aurait dû avoir un grand intérêt à prendre part ${ }^{20}$.

14 Puis, octobre, qui fut immédiatement envisagé en France par les organes de presse avec une grande inquiétude - y compris par L'Humanité. Aulard n'était pas en reste et le 14 novembre, il prit la plume pour faire part de la sienne : rappelant la défaite parallèle des Italiens et la nécessité de voler à leur secours, il dénonçait un "pacifisme outré et naïf », qui « répand plus de sang qu'une virile résistance armée à l'agression des ennemis de la paix. » Non content de cela, face à ce qui apparaissait désormais clairement comme une guerre civile, Aulard n'avait pas de doute : l'exemple que les Russes devaient suivre était celui de la France de 1793, déchirée elle-même par les conflits intestins, mais pas prête pour autant à céder sur ses frontières, sans avoir remporté d'abord le combat intérieur et ensuite celui contre les puissances réactionnaires. Pour cette raison, lui qui témoignait une réelle sympathie pour les partisans de l'organisation d'un gouvernement fort en Russie afin de continuer la guerre, excluait l'idée que les soviets soient à la solde du Kaiser, prenait soin de ne pas couper le fil avec les maximalistes de Lénine et souhaitait encore que l'issue des tensions internes retentissantes ne porterait pas préjudice à l'accord politique et militaire passé avec la France ${ }^{21}$.

15 Pourtant, quelques jours plus tard, le 25 novembre, quand les choses devinrent claires, et que le spectre d'une paix séparée parut prendre forme, Aulard se résolut à rédiger un autre article, qui ne fut pas publié - et pour cause - dans le Journal de Russie : avec des accents d'une grande dureté, il rappelait que «c'est la Russie même, que Lénine, le sachant ou ne le sachant pas, poignarde au cœur [...] Lénine n'est pas le peuple russe [...] et [...] malgré la notification officielle des ordres donnés par M. Lénine pour un armistice 
à tout prix, nous ne pouvons croire que cette trahison se consomme. » Et pourtant, alors que ce qui paraissait impossible semblait pourtant sur le point de réaliser, voilà qu'Aulard concluait ce même texte en rappelant comment les alliés auraient à subir les conséquences de cette trahison, parce que «ces prétendus pacifistes vont diminuer les chances de la paix dans le monde "; il avertissait que ce que Lénine accordait à son peuple était une reddition honteuse et non une paix honorable, parce que l'Allemagne n'aurait jamais consenti au rêve agraire des paysans russes. Bref, « la paix de Lénine, c'est la guerre, guerre civile, guerre étrangère, non seulement la honte, mais la servitude ${ }^{22}$. »

Ce qui explique sans doute la non-publication de cet article n'est pas tant la peur des représailles bolcheviques que la volonté du gouvernement de Paris de ne pas rompre l'ultime fil qui liait la France à la Russie. Dans les mois durant lesquels Trotsky conduisit les négociations de paix qui aboutirent au traité de Brest-Litovsk en mars 1918, la France en effet ne manqua pas de faire savoir à Lénine que s'il avait continué la lutte contre l'Allemagne, les alliés auraient non seulement aidé les bolchéviques d'un point de vue financier, mais auraient activement concouru à la reconstitution d'un « Etat russe fédéral et centralisé échappant à la domination allemande ${ }^{23}$. »

C'est pourquoi, les critiques d'Aulard, qui défendit toujours, par respect pour l'intérêt supérieur de la patrie, les choix de l'exécutif, tendirent à s'atténuer et qu'il en vint à son tour à soutenir les raisons en faveur d'un accord renouvelé entre la France et la Russie : dans un article du 24 décembre, il rappelait qu'en dépit de l'amertume causée par les choix pacifistes du nouveau gouvernement russe, en France, on tentait néanmoins de comprendre, on gardait confiance dans les pourparlers de l'envoyé Noulens avec Lénine, et on nourrissait même une certaine sympathie pour quelques-uns des motifs qui avaient donné lieu à la demande de paix des bolchéviques ${ }^{24}$. Dans un autre écrit du 4 janvier 1918, il plaidait la cause de la Société des nations, espérant que la Russie de Lénine ne prendrait pas ses distances avec le mouvement démocratique, abandonnerait le dogme de la lutte des classes et tiendrait ferme dans l'alliance avec les puissances de l'Entente ${ }^{25}$. Au lendemain du célèbre discours de Wilson de janvier 1918, Aulard reprit à nouveau la plume pour souligner à quel point le président américain voulait en tout état de cause inclure la Russie dans le nouvel ordre mondial qui devait naitre de la fin des hostilités, et pour rappeler que le peuple français voulait être aux côtés du peuple russe et il nourrissait alors encore l'espoir que l'entente n'en serait pas compromise ${ }^{26}$. Cependant, la brusque dissolution de l'Assemblée constituante interdit à Aulard de continuer dans cette voie : dans un autre article du 27 janvier, passant sous silence l'outrage à la souveraineté populaire, et toujours à la recherche d'un possible arrangement, Aulard entendait rappeler que la demande française de l'Alsace-Lorraine, au-delà des discours intéressés mis en circulation en Allemagne, était basée sur le véritable droit de chaque peuple à l'auto-détermination, et s'inscrivait par conséquent dans la droite ligne de l'esprit qui devait inspirer la Société des Nations - à laquelle il exhortait la Russie de participer ${ }^{27}$. Enfin, le 6 février, Aulard reprenait la parole pour souligner l'intérêt que l'on portait à la création d'une République fédérale en Russie : que les bolchéviques, attentifs lecteurs de 1793, ne devaient pas se méprendre sur le sens des événements liés au fédéralisme français pour en déduire que le peuple français leur était hostile. Au contraire, selon Aulard, la guerre civile qui eut alors lieu n'était pas tant fondée sur la forme institutionnelle de l'état, que sur la manière d'affronter la situation dramatique de la République à un moment où, aux Montagnards, fauteurs d'une dictature de la capitale (qui, selon lui, était nécessaire), s'opposaient les Girondins, qui entendaient gouverner 
dans le respect de la constitution. Il en était résulté un conflit marqué par les accusations de fédéralisme, parce qu'alors ce terme était synonyme de Contre-Révolution et que personne en France n'avait l'intention de renoncer à l'unité. Et pourtant, poursuivait Aulard, depuis ce temps-là, beaucoup d'eau avait coulé sous les ponts : en France, on regardait avec admiration les modèles politiques fédéraux américains et suisses, une école régionaliste bien vivante ne manquait pas d'exister, et il n'était pas possible de soulever cette question pour la simple raison qu'un état fédéral naissait spontanément et ne pouvait être construit artificiellement. En un mot, toujours selon Aulard, en France, on regardait favorablement l'entreprise colossale que l'on tentait en Russie et l'on était même convaincu que « le lien fédératif finira par s'établir, surtout si les révolutionnaires Russes empêchent l'empereur allemand de mettre la main sur leur Révolution ${ }^{28}$.»

Puis la paix de Brest-Litovsk mit un terme à tant de marques désinvoltes de confiance, qui avaient empêché Aulard d'exprimer ses propres critiques sur la Révolution bolchévique lesquelles au contraire émergent des observations contenues dans les conférences universitaires données entre temps ${ }^{29}$. Aulard, indigné par la trahison, n'aurait plus collaboré au Journal de Russie, mais aurait rapidement trouvé le moyen de s'intéresser encore à la Révolution de Lénine, puisqu'au lendemain de la conclusion du conflit, quand la ville de Paris était déjà devenue le point de rencontre de beaucoup d'exilés ${ }^{30}$, la Ligue des Droits de l'Homme décida de diligenter une enquête sur la situation politique en Russie : suite à ces témoignages, selon ce qu'en rapporte Furet, Aulard aurait manifesté une opposition frontale à l'ex-ministre du gouvernement révolutionnaire, Avksentiev, révélant son intérêt pour les positions des bolchévicks, justifiant la dictature

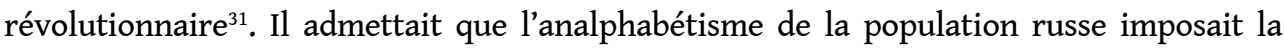
nécessité qu'une minorité guidât la politique révolutionnaire. Il continuait imperturbable à établir des rapprochements entre les soviets et les sections parisiens de 1789, et il se montrait insensible aux dénonciations de la Terreur que l'on disait répandues en Russie ; et toujours avec le regard tourné vers 1793, il concluait en imputant aux interventions militaires des Alliés, la dérive toujours plus violente engagée par la politique de Lénine. En somme, pour Aulard, la dénonciation d'une minorité bolchévique qui avait fait son lit de la démocratie politique, rappelait les nombreuses calomnies dont furent victimes les Montagnards en l'an II. Pour cette raison, il semblait soudainement incertain quant au jugement complexe à porter sur octobre. Et pourtant, la réponse d'Aksentiev, que Furet ne rapporte pas, dut lever ses doutes, puisque l'exilé Russe, s'adressant à lui avec le respect religieux de l'élève, reprenait à son compte l'analogie historique pour lui rappeler qu'il n'était en rien comparable à un émigré de Coblence et pour lui préciser que les bolchéviques n'avaient de commun avec les jacobins que le système terroriste ${ }^{32}$. On ne sait pas jusqu'à quel point les arguments d'Avksentiev firent mouche, puisque la Ligue des Droits de l'Homme aurait conclu son rapport en termes ambigus quant à la situation en Russie, en confirmant à la fois sa grande sympathie pour la Révolution et son profond regret pour sa dérive violente ${ }^{33}$. Pourtant, dans les mois suivants, les opinions d'Aulard semblent avoir changé, et ce qui le prouve, c'est une intervention de juillet 1919 dans laquelle il prend acte avec satisfaction de ce que les exilés Russes, pour lesquels il gardait cependant une grande sympathie, ne demandaient plus une intervention armée pour renverser les bolchéviques. Aulard considérait avec intérêt ce changement de stratégie, qui signifiait l'abandon des officiers blancs à eux-mêmes, puisqu'il était toujours plus convaincu que leur offensive en armes avait renforcé, plutôt qu'affaibli, Lénine. Selon lui, en fait, s'il y avait une possibilité que les bolchéviques soient contraints de céder leur prépondérance, cela ne pouvait venir que d'un coup de force militaire, étant donné que 
c'était bien l'armée qui, en raison de la guerre civile, s'était démesurément accrue, au point de constituer un élément susceptible de rétablir l'ordre dans le camp révolutionnaire. Encore ici, les références aux issues de la Révolution française sont tenues pour certaines : avec Lénine dans le rôle de Robespierre, les officiers blancs dans ceux des armées contre-révolutionnaires coalisées, et un futur général de l'Armée Rouge prêt à prendre les traits du jeune Bonaparte.

Cette approche des événements russes tend à expliquer pourquoi Aulard regardait désormais avec inquiétude toute éventuelle intervention armée contre les bolchéviques. Convaincu que leurs chances d'exporter la Révolution étaient minimes, et ne trouveraient de réel champ de manœuvre que par les carences d'une politique de démocratisation dans l'Europe issue de la guerre, Aulard insistait sur la nécessité d'affaiblir le front interne bolchévique, en ayant soin de ne pas enflammer le patriotisme russe; ceci explique pourquoi en octobre, dans la campagne contre l'intervention militaire et contre le blocus affamant le peuple russe, dans une lettre à L'Humanité, il s'exprimait en ces termes :

Je suis antibolcheviste, puisque je suis démocrate, et je le suis en tant que français. Je veux dire par là que ce serait folie d'appliquer à la France les méthodes d'un sanguinaire fanatisme oriental, tout comme ce serait folie de vouloir introduire chez nous le tsarisme. Mais laissons les Russes libres de bolcheviser ou de tsariser, si cela leur fait plaisir. D'ailleurs, pour les crimes qu'on attribue à Lénine et à Trotsky, soyons en état d'esprit critique. Robespierre et Danton, eux aussi, furent traités de monstres à face humaine, et l'Europe monarchique dénonçait la Révolution française comme une saturnale barbare. Cela ne veut pas dire que Lénine et Trotsky doivent être égalés, pour la valeur morale, à Danton et à Robespierre, ni que le bolchevisme soit une révolution constructive, comme l'a été la Révolution française. Cela veut dire qu'il faut se méfier des vérités officielles et que le peuple français ne doit pas traiter le peuple russe comme un peuple d'assassins. ${ }^{34}$

Si l'analogie historique continue à dominer les réflexions d'Aulard, il apparait ici clairement qu'il était parvenu à distinguer les modalités de l'action politique en fonction de l'environnement dans lequel elles se trouvent inscrites ; si l'inquiétude de se retrouver dans le rôle déplaisant de puissance étrangère lancée à l'assaut de la terre révolutionnaire le poussa encore à beaucoup douter de la violence léniniste, il lui semblait pourtant clair désormais que seule en France une révolution positive avait pu avoir lieu, et que la Russie, arriérée et à la population semi-analphabète, évoluait de toute évidence en ne faisant que poursuivre, même si c'était sous la forme révolutionnaire, sa propre tradition de barbarie orientale.

Par ailleurs, Aulard aurait répété ces mêmes considérations, à peine quelques semaines plus tard, quand, appelé à tracer un parallèle entre les Soviets et les comités révolutionnaires de la France de 1789, il aura soin de souligner qu'ils n'avaient rien en commun, puisque les soviets, qui constituaient le bastion de la dictature léniniste « n'ont aucun caractère démocratique, puisque ce ne sont pas tous les éléments populaires qui concourent à les former, mais seulement une minorité de travailleurs, surtout urbains, associés à quelques bourgeois plus ou moins déguisés en travailleurs ${ }^{35}$. » Ainsi, à partir du début des années 20, le parcours politique des deux Révolutions était tracé : l'une, héritant de la tradition culturelle du XVIII ${ }^{\mathrm{e}}$ siècle, même à travers les multiples difficultés pour la plupart générées par ceux qui s'opposaient à son cours, aurait conduit au triomphe de la liberté; l'autre - en s'implantant dans un tissu social dramatiquement arriéré - aurait seulement reproposé, et ce, même dans les termes de la pratique révolutionnaire, le dramatique continuum de la tradition politique de violence et de barbarie propre à la Russie moderne. 
22 Aulard aurait confirmé cette position, en commentant, en septembre 1920, les notes relatives au voyage en Russie des futurs leaders communistes Cachin et Frossard ${ }^{36}$. C'était en effet l'occasion de désapprouver avec sévérité leurs affirmations quant à la responsabilité de l'Entente dans l'échec de la réalisation du programme léniniste. En effet, Aulard contredisait ces affirmations en soutenant le fait que c'était précisément la guerre des puissances occidentales qui avait favorisé la tyrannie de Lénine et qui lui avait permis de se renforcer au pouvoir grâce à la vague patriotique qui avait secoué la société russe ${ }^{37}$. Position encore confirmée par Aulard au lendemain du célèbre discours de Lénine du 17 octobre 1920, par lequel il ajournait l'application du communisme : à cette occasion encore, tout en refusant un lien direct avec la révolution française, le portrait de Lénine en homme fort de la Russie, le seul capable de la gouverner en ayant recours dans le même temps à l'esprit patriotique et aux espérances de l'avenir, était encore une fois extrait du répertoire de 1793, puisqu'il ne manquait pas même un rapprochement avec le dernier grand discours parlementaire de Robespierre, à la veille de Thermidor ${ }^{38}$.

Les similitudes ne s'arrêtaient pourtant pas moins là, parce que sur la différence entre la révolution française et celle de Russie, Aulard, aurait toujours tenu ferme. Ainsi, au cours de l'année universitaire 1921-1922, dédiant son cours à la violence révolutionnaire, il aurait souligné combien l'apologie de la violence de la part des bolcheviks était peut-être :

utile pour pousser au progrès la masse ignorante et rurale du peuple russe. Peutêtre que la loi, dans ce milieu à demi-asiatique, la loi ne peut pas être l'expression de la volonté générale parce qu'il ne peut pas y avoir de volonté générale. Les bolcheviks pour justifier leur violence et leur dictature invoquent l'exemple de la révolution française, surtout l'exemple de la Convention nationale... c'est une erreur complète. Il n'y a pas un décret-loi sous la Révolution, par un seul... qui met la France au régime des décrets loi. Je ne veux pas dire que la violence n'ait pas joué un grand rôle dans la Révolution française, mais la Révolution française a été un grand effort pour supprimer la violence et l'anarchie d'Ancien Régime et pour établir une loi, la loi. On peut dire en gros que la Révolution française fut faite pour placer la France sous des lois, des vraies lois, uniformes pour tous les pays ${ }^{39}$.

Ces considérations n'auraient pu échapper à son rival de toujours, à savoir Albert Mathiez, entre-temps devenu communiste, qui, dans les colonnes de L'Humanité avait dénoncé le vieil historien de la Sorbonne comme n'ayant aucun sens de la notion historique de la légalité et argumentait à partir du postulat de base selon lequel celle-ci devait en toute occasion s'identifier à la démocratie parlementaire. S'ensuivait la liste détaillée des erreurs et des omissions d'Aulard, qui rendait évidente le fait que les positions du vieux professeur servaient de paravent à celles du Bloc national, lequel, et ce n'était pas un hasard, avait d'abord soutenu la contre révolution de Denikine et Kolchak et insistait à présent encore pour miser sur Wrangel, l'autre militaire blanc ${ }^{40}$.

L'affrontement était désormais sans issue et ce n'est pas un hasard si, peu de temps après, Aulard confirmait point par point l'idée que 1793 et octobre 1917 n'avaient rien à voir l'un avec l'autre : à l'occasion de la parution de L'Histoire des soviets, publiée à Paris en 1922, il mettait en garde contre les analogies, rappelant que « ces ressemblances ne sont que dans des formes, dans des détails, dans des gestes, dans des souvenirs de lecture. Essentiellement, la Révolution russe diffère autant de la Révolution française que la Russie diffère de la France ${ }^{41}$.» Dans cette optique, même la sympathie toujours manifestée par Aulard pour les révolutionnaires de février se colore d'amertume, en raison du caractère improbable de leur tentative : ceux-ci avaient réellement cru que la Russie pouvait devenir une démocratie en liant étroitement leur propre destin politique à 
celui des puissances occidentales; en mettant ce lien à la base de tout, ils avaient cependant oublié la profonde impréparation du peuple russe, qui ne les aurait pas compris dans leur effort noble et généreux de continuer la guerre, avec pour résultat, d'ouvrir la voie aux bolchéviks, qui auraient inversement fait un usage sage, bien qu'inapproprié, de la violence inhérente à la pratique politique russe ${ }^{42}$.

Au terme du parcours d'Aulard, se trouve le célèbre discours tenu en avril 1923 à la Sorbonne sur la signification de la violence dans l'épisode révolutionnaire français ${ }^{43}$. A l'encontre des appropriations abusives auxquelles les bolcheviks se livraient, Aulard rappelait que la théorie de la violence et de la dictature elle-même avaient été étrangères à la pratique révolutionnaire issue de 1789 , au point même que pendant la Terreur, inévitable conséquence de l'agression des puissances étrangères, l'esprit de la légalité avait soutenu le destin du nouvel ordre en France. Aucune lutte de classe, aucune dictature du prolétariat, n'avaient fait irruption dans les événements révolutionnaires français et c'était donc un procédé particulièrement prédateur (et absolument injustifié) que celui que les bolchéviks avaient rapidement mené, lorsqu'ils avaient tenté - par le biais du recours à l'analogie historique, par le biais de la création d'une légende de l'an II annonciatrice d'octobre - de donner une tradition historique à ce qui ne demeurait qu'une particularité qui leur était propre, produit de l'extraordinaire arriération de la société russe. Sur ce point, Aulard n'a jamais varié, renforçant encore sa propre conviction d'une impossible comparaison entre 1793 et 1917 dans l'article écrit au lendemain de la mort de Lénine. A cette occasion, il rappelait que :

Lénine n'était pas, il n'a jamais été un disciple de la révolution française, mais un disciple de Karl Marx, qui n'a jamais voulu étudier sérieusement l'histoire de notre Révolution, et qui s'est appliqué à l'éliminer, comme bourgeoise. A la place de la Révolution française, c'est la Commune de Paris de 1871 qu'il installa dans l'imagination du peuple, comme un précédent et un symbole de la révolution socialiste et prolétarienne. Non pas, certes, la vraie Commune de Paris qui, issue d'une révolte du patriotisme et d'une fièvre obsidionale, ne devint que peu à peu socialiste et ouvrière ; mais d'une Commune de Paris imaginaire avec fort peu de traits réels ${ }^{44}$.

Son texte, traduit en russe par l'exilé Boris Mirkine-Guetzevich, qui, entre temps, avait commencé à collaborer avec Aulard à la Société d'histoire de la Révolution française, aurait provoqué - comme l'a révélée T. Kondratieva - la riposte violente des historiens soviétiques, qui ouvrirent un feu nourri contre ce qui apparaissait comme une authentique provocation sous la forme d'au moins quatre articles entre 1924 et $1927^{45}$. L'extrême dureté de cette riposte s'explique aussi par le fait qu'Aulard, qui ne faisait plus désormais mystère de son soutien à la dissidence russe en exil ${ }^{46}$, avait confirmé point par point sa propre position, jusqu'à rappeler, à l'occasion de la reprise des relations diplomatiques, la nécessité pour la Russie de tourner ses regards sur l'exemple révolutionnaire français ${ }^{47}$. Mais c'était là désormais un dialogue de sourds, semblable à celui qui semblait avoir lieu dans les réunions organisées par la Ligue des Droits de l'homme durant l'année 1926 pour dénoncer la menace fasciste : jamais, au cours de ces rencontres, Aulard ne voulut accepter le soutien des communistes français, leur reprochant d'être un élément anti-démocratique, et donc, substantiellement, tout à fait semblable au fascisme montant. En rappelant que «si les bolchévistes l'emportaient en France, la liberté ferait les frais de leur victoire, tout comme si c'étaient les fascistes qui l'emportaient", Aulard rendait caduque toute possibilité de dialogue avec le Parti Communiste Français dont il dénonçait à maintes reprises le sectarisme ${ }^{48}$. A la veille de sa mort, il écrivait encore un dernier article dans lequel il soulignait comment octobre avait 
transformé la Révolution d'un moment d'extraordinaire liberté en un espace où le citoyen perdait tous ses droits et n'acquérait que des devoirs face au nouvel Etat soviétique ${ }^{49}$. Entre-temps, les querelles historiographiques n'en avaient pas moins rebondi sur le terrain politique, et beaucoup conditionné l'action du nouveau-né Parti Communiste Français. Dès 1923, il y avait l'interdiction de rester dans la Ligue des droits de l'Homme (ainsi que dans la franc-maçonnerie ${ }^{50}$ ), et dès 1924 , la lutte soutenue contre le cartel de gauche qui vit ainsi revenir au pouvoir ce monde radical-socialiste bien représenté précisément par la personnalité d'Aulard ; enfin, sur le plan historiographique, il y aurait eu la consécration définitive de Mathiez, qui justement dans les années postérieures à 1923, aurait liquidé Danton et imposé le retour en grâce du robespierrisme dans les études révolutionnaires ${ }^{51}$. En somme, la boucle était bouclée : et aux thèses d'Aulard sur l'impossibilité d'un quelconque rapprochement entre la Révolution française et celle de Russie, sans pour autant remettre en cause le binome 1776-1789, d'autres thèses totalement opposées suivirent, toutes fortement imprégnées du mythe d'octobre, à la base desquelles l'analogie historique valait au contraire comme preuve avérée de la linéarité du processus historique et donc, légitimait le caractère inévitable de la conclusion, dans le sens soviétique, du processus révolutionnaire qui n'avait été qu'esquissé par la France jacobine.

\section{NOTES}

1. René SIDERSKY, Confidences révolutionnaires, Paris, Clavreuil, 1967, p. 6-15.

2. James FRIGUGLIETTI, Albert Mathiez, historien révolutionnaire, 1874-1932, Paris, Société des Etudes Robespierristes, 1974, p. 183-198.

3. Humanité, 24 octobre 1928, p. 2. La référence à Henry Beraud, s'inscrit dans les termes de la prise de distance qu'Aulard prit avec l'ouvrage de ce dernier intitulé, Ce que j'ai vu à Moscou, Paris, Editions de France, 1925, où, en critiquant par leurs positions libertaires les bolcheviques, il rappelait comment «la vraie révolution russe, la preneuse de la Bastille, c'est celle de février, celle qui renversa le tsarisme, et non point celle qui jugula, les libertés publiques ». Les critiques de l'historien à l'égard de Béraud, se trouvent dans Alphonse AULARD, Soyons justes et vrais, dans Le Quotidien, 13 décembre 1925. Marc Nouschi évoque cette participation d'Aulard au journal Le Quotidien, dans «Georges Boris, analyste de la crise économique : le réformisme et sa pratique dans les années 1930 en France ", Le Mouvement social, n 115 (avril-juin 1981), p. 51.

4. Au sujet du dépôt du fonds Aulard à Harvard, voir Harvard University, Report of the President of Harvard College and reports of departments, 1931-1932, p. 231: "The Library of Alphonse Aulard, French historian, 2328 volumes and 1213 pamphlets of the French revolution, given in memory of Archibald Cary Coolidge ». La cote d'archives à la donation est dans Harvard Archives, Hug. Fp. 22. 8 box 1, Gift number 1224, date Oct 10, 1932. La cote prouvant que le donateur anonyme est précisément James Buell Munn est à l'inverse : ivi, UA, III, 50.15.70.4, Accession book, 1932, Oct 10, 1932 : Aulard Collection, Anonymous [J.B. Munn, New York].

5. Voir les réflexions consacrées au sujet par Georges. BELLONI, Aulard, historien de la Révolution, Paris, xxx, 1949, p. 140-148 et dans l'introduction à son travail, celles d'Albert Bayet, gendre d'Aulard, dans lesquelles, incidemment, il se souvient d'abord que le grand historien aurait 
«montré de la sympathie au Parti communiste naissant» (p. XII), et ensuite, qu'il aurait même salué « comme un immense espoir humain la Révolution soviétique » (p. XV).

6. Sergio LuzzATo, La Marsigliese stonata. La sinistra francese e il problema storico della guerra giusta, 1848-1948, Bari, Dedalo, p. 44-48.

7. François FURET, Le passé d'une illusion. Essai sur l'idée communiste au $20^{e}$ siècle, Paris, Laffont, 1995.

8. Raoul LABRY, Autour du bolchevisme, La Roche-sur-Yon, l'Auteur, 1921.

9. Henri DE MAN, Au-delà du marxisme, Bruxelles, l'Eglantine, 1927.

10. L'ensemble de la documentation citée par la suite est conservée à l'université d'Harvard, Houghton Library, Ms. Fr. 46, Cours d'Alphonse Aulard à la Sorbonne (1886/7-1921/2), vol. 33 et 35 en particulier.

11. Voir dans Alphonse AULARD, Solidité de l'amitié française, ivi, vol. 33 et dans Journal de Russie (JR), $n^{\circ}$ 43, 23 novembre (6 décembre) 1917.

12. Sur la manifestation du $1^{\mathrm{er}}$ avril, voir Sergio LUzzATo, op. cit., p. 44-45, ainsi que Georges MICHON, L'alliance franco-russe, 1891-1917, Paris, Delpeuch, 1927, p. 277-278.

13. Voir le discours de Aulard dans Bulletin officiel de la Ligue des Droits de l'Homme, 1917, p. 414-418; le texte, ensuite traduit en russe est en revanche????? Alphonse AULARD, La révolution française et la révolution russe. Lettre aux citoyens de la libre Russie, Lausanne, Payot, 1917. Sur ce point, voir quelques notes de Sophie coEURÉ, La grande lueur à l'Est. Les Français et l'Union Soviétique, 1917-1939, Paris, Seuil, 1999, p.26. Cette auteure fournit ensuite un témoignage intéressant de cette initiative éditoriale, dans un article rédigé quelques années plus tard, à l'occasion de la disparition du dessinateur Willette, qui devait préparer le croquis de la couverture de la brochure. Voir Alphonse AULARD, « Willette et la révolution russe ", Le populaire de Nantes, 10 février 1926.

14. Hubert BOURGIN, Le parti contre la patrie, Paris, Plon, 1924, p. 211 : «Ce meeting fut transformé par la partie

15. Voir le jugement cinglant sur octobre de Ludovic NAUDEAU, En prison sous la terreur russe, Paris, Hachette, 1920, p. VI : «minorité séditieuse qui, en novembre 1917, profitant de l'immense détresse d'un peuple en désarroi, s'empara du pouvoir par un pronunciamento à la mexicaine ».

16. Raoul LABRY, «Comment nous avons été renseignés sur la Russie », Mercure de France, 1920, n -522, p. 667-669.

17. Ludovic NAUDEAU, op. cit., dans JR, $n^{\circ} 35,2$ (15) novembre 1917.

18. Alphonse AULARD, « France et Russie », JR, n 35, 2 (15) novembre 1917.

19. Alphonse AULARD, «Solidité de l'amitié », op. cit.

20. Alphonse AULARD, «Esprit démocratique en France», Cours, vol. 33, et $J R, \mathrm{n}^{\circ} 55$, 16 (29) décembre 1917.

21. Alphonse AULARD, "Angoisse et sympathe », ibid, vol.33, publié sous le titre "Angoisse et sympathie et confiance ", JR, $n^{\circ}$ 60, 23 décembre 1917 (5 janvier 1918).

22. Alphonse AULARD, Emotion, indignation, dans Cours, vol. 33.

23. Sur ce point, voir Kalervo Hovi, Cordon sanitaire or barriere de l'Est? The emergence of the New French Eastern European Alliance policy, 1917-1919, Turku, Turun Vliopisto, 1975, p. 92; Michael Jabara CARLEY, "The origins of the French intervention in the Russian civil war, januarymay 1918: a reappraisal », Journal of modern history, 48 (1976), p. 413-439 et « The politics of antibolshevism: the French government and the russo-polish war, December 1919 to may 1920 ", Historical Journal, 19 (1976), p. 163-189, ainsi que, plus récent, Anne HOGENHUIS-SELIVERSTOFF, « La trace ténue d'une alliance ancienne. La France et la Russie, 1920-1922 », dans Guerres mondiales et conflits contemporains, PUF, $\mathrm{n}^{\circ} 193,1999, \mathrm{p} .118-119$, en particulier

24. Alphonse AULARD, ce qu'on pense de la Russie à Paris, Cours, vol.33, et JR, $\mathrm{n}^{\circ} 73$, 17 (30) janvier 1918.

25. Alphonse AULARD, Et la société des nations?, JR, n² 2, n. s., 21 (8) mars 1918. 
26. Alphonse AULARD, La révolution russe et le président Wilson, Cours, vol. 33, et $J R, \mathrm{n}^{\circ} 52$, 28 (15) mai 1918 (au sujet du décalage entre la rédaction et la publication, voir la note introductive de la rédaction (redazione) : «Nous tenons à publier, malgré son ancienneté, étant donné l'intérêt qui s'attache à la question, l'article ci-après de notre éminent collaborateur, Alphonse Aulard »).

27. Alphonse AULARD, La question de l'Alsace-Lorraine, Cours, vol. 33, et JR n 54, 30 (17) mai 1918.

28. Alphonse AULARD, La République fédérative, Cours, vol. 33, et $J R, \mathrm{n}^{\circ} 35, \mathrm{xxxxx}$ date .

29. En décembre 1917, rappelant le précédent de 1762, qui obligea la France à faire la paix, Aulard déclarait en chaire : «notre espérance est que la Russie ne devienne pas un relais allemand. Cela dépendra de la future constituante... », tandis que dans les premiers jours de 1918, à l'occasion de l'arrestation du ministre roumain à Petrograd, il trouvait bon de rappeler combien la Révolution française avait à l'inverse toujours pleinement respecté les ambassadeurs. Voir ses notes dans Cours, leçons du 3 décembre 1917 et du 5 janvier 1918.

30. Robert H. JoHnson, New Mecca, new Babylon. Paris and the Russian exiles, 1920-1945, Montréal, Mc Gill University Press, 1988.

31. François FURET, op. cit., p. 80-81.

32. «Votre parallèle entre la révolution française et la nôtre ne me paraît pas exact. Laissez-moi vous dire que nous ne sommes pas des émigrés de Coblence. Nous avons été expulsés... Laissez le bolchevisme se développer tel qu'il est et sans intervenir et vous verrez que, las d'anarchie, le peuple abattu, épuisé, enfin désespéré, finira par accepter, par implorer n'importe quel « ordre ». Quant au parallèle que vous avez fait sur les minorités agissantes, permettez-moi de vous répondre que ce n'est pas sur l'élite du peuple que les bolchéviks s'appuient. Au contraire, ils cherchent à détruire ce qui est intellectuel. Faites attention, M. le professeur, ce sont les professeurs qu'ils tuent. Et en outre, il y a une différence énorme entre vos jacobins et nos bolchéviks. Laquelle? Le terrorisme est leur seul trait commun ", op.cit., dans Christian JELEN, L'aveuglement. Les socialistes et la naissance du mythe soviétique, Paris, Flammarion, 1984, p. 56-57.

33. Cette initiative aurait pourtant coûté à la Ligue des Droits de l'Homme les rares sympathies de la gauche radicale. Voir les considérations à ce propos de Georges Michon, op. cit., p. 293, qui en parlant des trop grandes erreurs commises par la France à l'égard de la Russie soviétique, rappelle aussi « la singulière enquête de la Ligue sur le bolchevisme ».

34. L'Humanité, 26 octobre 1919.

35. Alphonse AULARD, «Les soviets et la Révolution française », Floréal, 1920, p. 77.

36. Boris sокоцоv, Le voyage de Cachin et de Frossard dans la Russie des Soviet: faits et documents, Paris, Povolozky, 1920.

37. Alphonse AULARD, "Le pèlerinage de MM. Cachin et Froissard», dans Ere nouvelle, 13 septembre 1920.

38. Alphonse AULARD, « Mélancoliques aveux de Lénine », ivi, 16 novembre 1921.

39. Dans Cours, vol. 35, leçon du $1^{\mathrm{er}}$ mars 1922.

40. Albert MATHIEZ, « M. Aulard et la légalité », dans L'Humanité, 21 mars 1922.

41. Alphonse AULARD, «Deux révolutions, 1789-1917 », dans Henri DE WEINDEL (dir.), Histoire des Soviets, Paris, Jacques Makowsky, mai 1922, p. 9-12 (p. 10).

42. «Or, ce sont des socialistes patriotes qui prirent en main le gouvernement après la chute du tsar. Amis et disciples de la Révolution française, ils voulurent, non seulement continuer la défense nationale, mais la rendre plus active, faire un gigantesque effort pour la victoire. Ils ne comprirent pas le peuple russe, et le peuple russe ne les comprit pas. On va lire le récit de leur échec dans les opérations militaires et civiles, les résultats du malentendu entre le peuple et eux. Il nous semble, à nous français, qui sommes pénétrés de gratitude et d'admiration pour ces hommes généreux, qu'ils avaient raison, que la démocratie russe ne pouvait se fonder qu'en unissant son sort aux démocraties occidentales, en empêchant que ces démocraties ne fussent 
écrasées par le militarisme allemand. La défection russe, au temps de Lénine, nous coûta affreusement cher, et nous aurions peut-être du fait de cette défection et sans l'intervention américaine, perdu la guerre, ce qui aurait probablement amené le rétablissement du tsarisme en Russie. Mais ce pauvre peuple n'en pouvait plus et d'autre part, il était, il est très ignorant ", ivi, p. 11.

43. Voir à cet égard, Pierre SERNA, «La République et le coup d'état. Les crises de la III République et la hantise du 18 brumaire ", Politix, 39, 1997, p. 149. Le texte d'Alphonse Aulard, intitulé « La théorie de la violence et la Révolution française », est reproduit dans Etudes et leçons sur la Révolution française, neuvième série, Paris, Alcan, 1924, p. 1-27. Voir en outre la synthèse, du même titre, parue dans la Dépêche de Toulouse, 13 avril 1923.

44. Alphonse AUlARD, "Lénine et les droits de l'homme", Cahiers des droits de l'homme, 10 février 1924, p. 52.

45. Tatiana KondRATIEVA, Bolcheviks et jacobins: itinéraire des analogies, Paris, Payot, 1989, p. 216-221.

46. En septembre 1924, Evguéni Guéguétschkari, ministre des affaires étrangères du gouvernement de Géorgie en exil, s'adressait en effet à lui avec déférence, parce que dans les colonnes des journaux dans lesquels le grand historien avait coutume de collaborer, on mettait en lumière la répression dramatique qui était en cours dans la République envahie par les troupes soviétiques.

47. Alphonse AULARD, « Proposons aux Soviets l'exemple de la Révolution française », Le Quotidien, 18 janvier 1925.

48. Alphonse AULARD, «Bolchevisme et fascisme », Le populaire de Nantes, 19 août 1926.

49. Alphonse AULARD, « La liberté politique », Révolution française, 81, 1928, p. 366-378.

50. Henri See, Histoire de la ligue des Droits de l'Homme, 1896-1926, Paris, Ligue des Droits de l'Homme, 1927, p. 176-178.

51. James FRIGUGLIETTI, op. cit., p. 182-204.

\section{RÉSUMÉS}

En s'appuyant sur les notes et les manuscrits d'Alphonse Aulard, conservés à la bibliothèque Houghton de Harvard, l'article passe en examen combien l'interprétation de 1789 représentait, pour le grand historien de la révolution française, une clé de la lecture de celle de 1917. Dans le climat patriotique de la Grande Guerre, Aulard aurait tout d'abord manifesté enthousiasme pour la révolution de février pour s'inquiéter face à la montée au pouvoir des bolcheviques et leur reprocher rapidement le choix de la paix avec l'Allemagne. C'est pourquoi, dans l'après-guerre français, la montée du mouvement communiste était destinée à rapidement effacer sa lecture historique (et politique) de la révolution française.

Largely based on Alphonse Aulard's manuscripts held in the Houghton Library at Harvard, the article shows how much his interpretation of the French Revolution was pivotal in considering the Russian Revolution of 1917. In the patriotic climate of the World War I, Aulard was enthusiastic at the news of the Russian Revolution in February, but he was then cautious in front of the insurrection of October and he finally refused the Bolshevik power because it entailed the peace with Germany. However, after the World War I, in France, Aulard's anti-Bolshevik 
positions were the main polemical argument to put an end to his historical (and political) interpretation of the French Revolution.

INDEX

Keywords : Alphonse Aulard, Historiography, French Revolution, Russian Revolution, Comparative revolutions

Mots-clés : Alphonse Aulard, Révolution française, Révolution russe, historiographie, révolutions comparées

\section{AUTEUR}

\section{ANTONINO DE FRANCESCO}

Université de Milan

Traduction Virginie MARTIN

Université Paris 1 Panthéon-Sorbonne 